\title{
Monopole effects on Polyakov loop and its gauge independence in QCD
}

\author{
Yoshimi Matsubara $^{\mathrm{a} *}$, Sawut Ilyar ${ }^{\mathrm{b}}$, Tsuyoshi Okude $^{b}$, Kenji Yotsuji $^{b}$ and Tsuneo Suzuki ${ }^{b}$ \\ ${ }^{a}$ Nanao Junior College, Nanao, Ishikawa 926, Japan \\ bepartment of Physics, Kanazawa University, Kanazawa 920-11, Japan
}

Monte-Carlo simulations of abelian projection in $T \neq 0$ pure lattice QCD show that 1) Polyakov loops written in terms of abelian link fields alone play a role of an order parameter of deconfinement transition, 2) the abelian Polyakov loops are decomposed into contributions from Dirac strings of monopoles and from photons, 3) vanishing of the abelian Polyakov loops in the confinement phase is due to the Dirac strings alone and the photons give a finite contribution in both phases. Moreover, these results appear to hold good in unitary gauges. This suggests that monopole condensation as the color confinement mechanism is gauge independent.

\section{Introduction}

It is one of the most important subjects to understand mechanism of color confinement in QCD. The abelian projection of QCD is to extract an abelian theory with charges and monopoles performing a partial gauge-fixing. 'tHooft conjectured an interesting idea [1] that condensation of the abelian monopoles produces color confinement due to the dual Meissner effect.

There are infinite ways of extracting an abelian theory out of QCD. Maximally abelian(MA) gauge is exciting. In this gauge, many interesting facts have been found. There are phenomena called abelian dominance [2]. The string tension from abelian Wilson loops is produced only by monopoles 3 3. 5]. The effective monopole action is derived from vacuum configulations [6]. In the infinite volume limit, entropy dominance over energy induces the condensation of the monopoles, which leads us to the confinement of the abelian charges ,i.e., the color confinement. Therefore the (extended) monopoles control the confinement mechanism in MA gauge.

The following subjects are to be clarified:

1) relation of the monopole dynamics to the deconfinement transition and

2) gauge dependence of the monopole dynamics.

From this point of view, we investigate the

\footnotetext{
*Presented by Y.Matsubara.
}

Polyakov loop which is an important order parameter of the deconfinement transition.

In this note, we show the abelian Polyakov loop is written by a product of monopole Dirac string part and photon part and vanishing of the abelian Polyakov loop comes only from the monopole Dirac-string contributions not only in MA gauge but also in unitary gauges.

\section{Abelian Polyakov loop and monopole Dirac-string}

We adopt the usual $S U(2)$ and the $S U(3)$ Wilson actions. To study gauge dependence, we consider three types of abelian projection. The matrices to be diagonalized are $\sum_{\mu}\left[U(s, \mu) \sigma_{3} U^{\dagger}(s, \mu)+U^{\dagger}(s-\hat{\mu}, \mu) \sigma_{3} U(s-\hat{\mu}, \mu)\right]$ in MA gauge, Polyakov loop in Polyakov gauge and plaquette in $F_{12}$ gauge.

After any gauge fixing is over, we can extract an abelian link variable $u(s, \mu)$ and an angle variable $\theta_{\mu}(s)$ from it 3 .

Now let us show that an abelian Polyakov loop operator of an external current $J_{4}(s)$

$P=\operatorname{Re}\left[\exp \left\{i \sum_{s^{\prime}=s}^{s+\left(N_{4}-1\right) \hat{4}} J_{4}\left(s^{\prime}\right) \theta_{4}\left(s^{\prime}\right)\right\}\right]$,

is given by a product of monopole and photon contributions.

Using the definition of a plaquette variable 


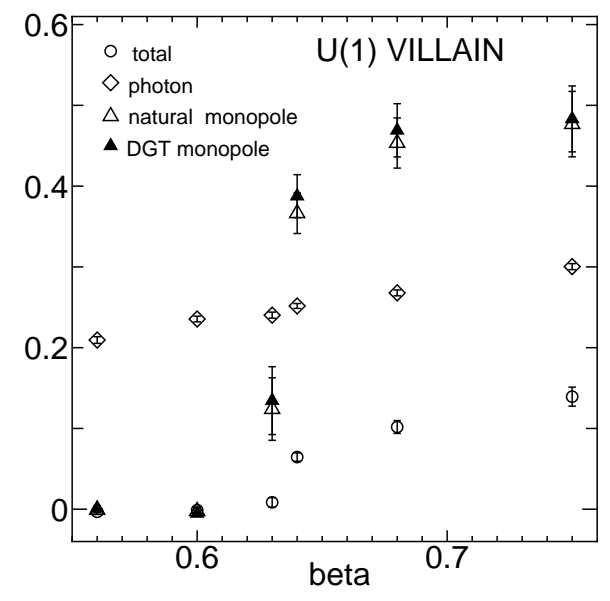

Figure 1. Monopole Dirac string and photon contributions to Polyakov loops in the Villain model of compact QED.

$f_{\mu \nu}(s)=\partial_{\mu} \theta_{\nu}(s)-\partial_{\nu} \theta_{\mu}(s)$, we get

$\theta_{4}(s)=-\sum_{s^{\prime}} D\left(s-s^{\prime}\right)\left[\partial_{\nu}^{\prime} f_{\nu 4}\left(s^{\prime}\right)+\partial_{4}\left(\partial_{\nu}^{\prime} \theta_{\nu}\left(s^{\prime}\right)\right)\right]$,

where $D\left(s-s^{\prime}\right)$ is the lattice Coulomb propagator.

Since $\partial_{4}^{\prime} J_{4}(s)=0$, the second term in the righthand side of (2) does not contribute to the abelian Polyakov loop (11).

The plaquette variable can be decomposed into three terms:

$$
\begin{aligned}
f_{\mu \nu}(s)= & -\epsilon_{\mu \nu \alpha \beta} \partial_{\alpha}^{\prime} C_{\beta}(s) \\
& +\partial_{\mu} \bar{\theta}_{\nu}(s)-\partial_{\nu} \bar{\theta}_{\mu}(s)+2 \pi n_{\mu \nu}(s)
\end{aligned}
$$

which $n_{\mu \nu}(s), C_{\mu}$ and $\bar{\theta}_{\mu}$ are an integer-valued Dirac strings [8], a dual potential and a photon terms, respectively. The dual potential term has no contribution because of the antisymmetric tensor. Therefore we have $P=\operatorname{Re}\left[P_{1} \cdot P_{2}\right]$ where

$$
\begin{aligned}
P_{1}= & \exp \left\{-i \sum_{s^{\prime}=s}^{s+\left(N_{4}-1\right) \hat{4}} J_{4}\left(s^{\prime \prime}\right)\right. \\
& \left.\sum_{s^{\prime \prime}} D\left(s^{\prime}-s^{\prime \prime}\right) \partial_{\nu}^{\prime}\left(\partial_{\nu} \bar{\theta}_{4}\left(s^{\prime \prime}\right)-\partial_{4} \bar{\theta}_{\nu}\left(s^{\prime \prime}\right)\right)\right\}, \\
P_{2}= & \exp \left\{-2 \pi i \sum_{s^{\prime}=s}^{s+\left(N_{4}-1\right) \hat{4}} J_{4}\left(s^{\prime}\right)\right.
\end{aligned}
$$

$$
\left.\sum_{s^{\prime \prime}} D\left(s^{\prime}-s^{\prime \prime}\right) \partial_{\nu}^{\prime} n_{\nu 4}\left(s^{\prime \prime}\right)\right\}
$$

We observe separately the real parts $P_{p}$ and $P_{m}$ of the photon $P_{1}$ and the Dirac-string $P_{2}$, respectively.

\section{Measurements}

\subsection{The Villain form of QED}

We first check that the separation works well in Villain form of the compact $U(1)$ [7] on a $8^{4}$ lattice. Since there are natural monopoles and DeGrandToussaint(DGT) monopoles in the Villain case of QED, we observe $P_{m}$ in terms of the two types of the monopoles. The results are shown in Fig. 1.

1)The monopole Dirac-string data vanish in the confinement phase, whereas the photon data remain finite and change gradually for all $\beta$. The characteristic features of the Polyakov loops are then due to the behaviors of the Dirac-string contributions alone.

2)Monopole Polyakov loops show more enhancement than the total ones for $\beta>\beta_{c}$.

3 Both types of monopoles give almost the same results.

\subsection{The MA gauge in $S U(2)$ and $S U(3)$ QCD}

The Monte-Carlo simulations were done in $S U(2)$ on $16^{3} \times 4$ lattice $(\beta=2.1 \sim 2.5)$ in the MA and the unitary gauges. In $S U(3) \mathrm{QCD}$, we adopted $10^{3} \times 2$ lattice $(\beta=5.07 \sim 5.12)$. All measurements were done every 50 sweeps (40 sweeps in the $S U(3)$ case) after a thermalization of 2000 sweeps. We took 50 configurations totally for measurements.

1)We plot the $S U(2)$ data in the MA gauge in Fig. 2. The abelian Polyakov loops remain zero in the confinement phase, whereas they begin to rise for $\beta>\beta_{c}=2.298[9]$. The monopole Dirac string contribution is zero for $\beta<\beta_{c}$, whereas it begins to rise rapidly and it reaches $\sim 1.0$ for large $\beta$. On the other hand, the photon part has a finite contribution for both phases and it changes only slightly. Characteristic behaviors of the abelian Polyakov loops are then explained by the Diracstring part of monopoles alone.

2)The same results are obtained also in pure 


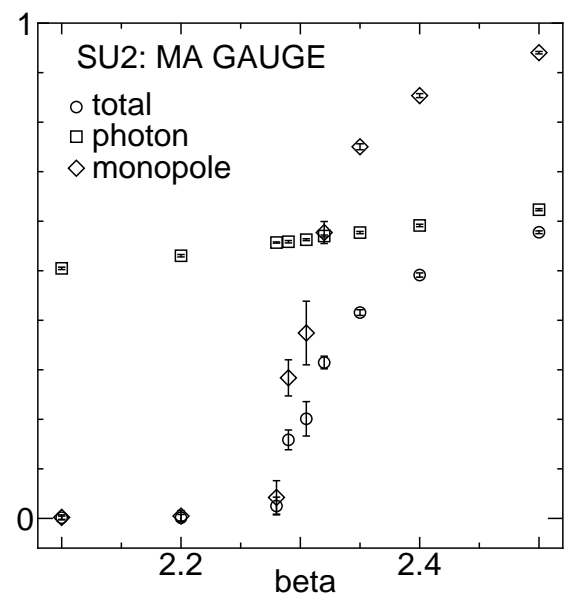

Figure 2. Monopole Dirac string and photon contributions to Polyakov loops in the MA gauge in $S U(2)$ QCD.

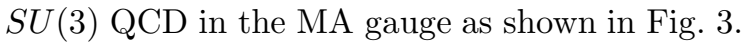
There is a clear hysteresis behavior showing the first order transition.

\subsection{The unitary gauges}

The data in the Polyakov gauge are plotted in Fig. 国. It is very interesting to see that the abelian and the Dirac-string Polyakov loops are zero in the confinement phase, which suggests occurrence of flux squeezing in the unitary gauge, too. They show finite contribution above the critical temperature $\beta_{c}$. Photon contributions are finite and change gradually in both phases. In the case of $F_{12}$ gauge, the results are very similar to these in Polyakov gauge.

\section{Conclusion}

Our analyses done here show that abelian monopoles are responsible for confinement in $S U(2)$ and $S U(3) \mathrm{QCD}$, and condensation of the monopoles is the confinement mechanism. These are the first phenomena suggesting gauge independent realization of the 'tHooft conjecture.

\section{REFERENCES}

1. G.'tHooft, Nucl.Phys.B190,(1981)455.

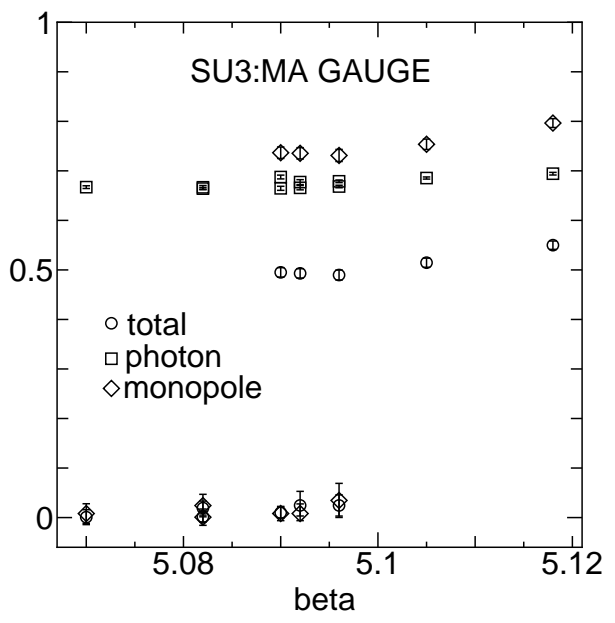

Figure 3. Monopole Dirac string and photon contributions to Polyakov loops in the MA gauge in $S U(3)$ QCD.

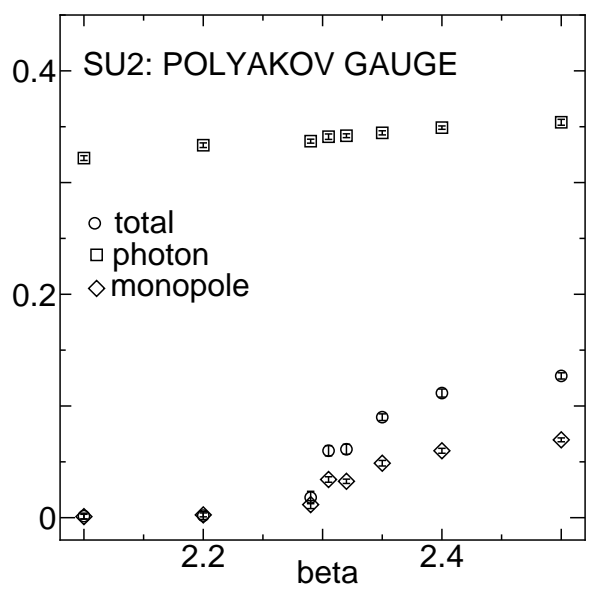

Figure 4. Monopole Dirac string and photon contributions to Polyakov loops in the Polyakov gauge. 
2. T.Suzuki, Nucl.Phys.B(Proc. Suppl.) 30, (1993) 176 and see also references therein.

3. H.Shiba and T.Suzuki, Phys.Lett.B333, (1994)461.

4. S.Ejiri,S.Kitahara,Y.Matsubara and T.Suzuki, Kanazawa University Report KANAZAWA 94-14, 1994.

5. J.D.Stack, S.D.Neiman and R.J.Wensley, Phys. Rev.D50,(1994)3399.

6. H.Shiba and T.Suzuki, Nucl.Phys.B(Proc. Suppl.)34,(1994)182 .

7. J.Villain, J.Phys.(Paris)36,(1975)581.

8. T.A.DeGrand and D.Toussaint, Phys. Rev.D22,(1980)2473.

9. J.Engels, J.Jersak, K.Kanaya, E.Laermann, C.B.Lang, T.Neuhaus and H.Satz, Nucl.Phys.B280,(1987) 577. 